\title{
Influence of steam sterilization induced surface changes of polyester materials on its biocompatibility
}

\author{
P D NAIR* ${ }^{*}$ P J DOHERTY ${ }^{\dagger}$ and D F WILLIAMS ${ }^{\dagger}$ \\ Sree Chitra Tirunal Institute for Medical Sciences and Technology, Thiruvananthapuram \\ 695012. India \\ ${ }^{\dagger}$ Department of Clinical Engineering, University of Liverpool, UK \\ MS received 14 December 1994; revised 6 August 1997

\begin{abstract}
Steam sterilization is conventionally used for the sterilization of materials used in surgery and medicine. When subjected to repeated steam sterilization, oligomers of polyethylene terephthalate are produced on the surface of the polyester materials. The consequences of the production of such oligomers on the biocompatibility of the polyester material have been investigated. Growth inhibition of L929 fibroblast cells has been observed on the repeatedly sterilized materials in the in vitro cell-material contact studies. In vivo implantation of the repeatedly sterilized materials elicited a hostile tissue response comprising enhanced macrophage activity, which could have an adverse effect on the long term stability of the polyester materials.
\end{abstract}

Keywords. Steam sterilization; polyester; surface; oligomer; biocompatibility.

\section{Introduction}

Steam sterilization by autoclaving has traditionally been the most widely used method for medical instruments. The process should be used with caution, while sterilizing polymers, as both heat and steam can drastically alter the properties of materials. The main deleterious effect arising due to steam sterilization is while the hydrolysis of the polymer takes place. Haberman and Waitzova (1985), observed the release of contaminating substances when PVC containing aqueous suspensions were autoclaved. Seemingly stable polymers can also undergo changes in surface morphology especially if the glass transition temperature of the polymers is exceeded while autoclaving. Conway et al (1979) in a scanning electron microscopy study of different types of arterial prostheses observed a layer of oligomers on autoclaving, which resulted in an increase of haemolysis. Studies of Nair and Srinivasan (1984) and Nair (1990) on the effect of steam sterilization of polyester fabrics used in medical applications, indicated that the cyclic trimer oligomer increased on repeated autoclaving. The present study reports the consequences of the oligomer formation on the biocompatibility of the polyester materials.

\section{Materials and methods}

\subsection{Materials}

Polyethylene terephthalate (PET) films (Garware, India) were used after soxhlet extraction of the films by methanol, water and ether to remove residual oligomers, sizing and finishing agents. The cleaned PET films were further wiped with methanol

*Author for correspondence 
before packing for steam sterilization. The samples were steam sterilized by autoclaving for $15 \mathrm{~min}$ at $121^{\circ} \mathrm{C}, 137.9 \mathrm{kNm}^{-1}$ pressure and subjected to repeated sterilization cycles of 30 and $60 \mathrm{~min}$.

\subsection{Cell line}

Primary cell line, L929 fibroblast was maintained at $37^{\circ} \mathrm{C}$ in Eagle's medium 199 (Gibco) supplemented with $5 \%$ foetal calf serum (Gibco) and 1\% antibiotic-antimycotic solution in an incubator with $5 \% \mathrm{CO}_{2} / 95 \%$ air atmosphere. The cell line was detached when required using trypsin-EDTA (Gibco) in phosphate buffered saline. The detached cells were further grown as a monolayer to a cell count of $1 \times 10^{5}$ after $72 \mathrm{~h}$.

\subsection{Cytotoxicity}

2.3a Indirect contact test: The cytotoxicity of aqueous extracts of the PET films after the different sterilized cycles was assessed using a 3-(4, 5 dimethyl thiazol-2-yl)-2, 5 diphenyl tetrazolium bromide, (MTT) assay (Nair et al 1997). The aqueous extracts of PET films were prepared by immersing $50 \times 10 \mathrm{~mm}$ strips in $10 \mathrm{ml}$ of sterile water at $80^{\circ} \mathrm{C}$ for 2 weeks.

MTT assay: The MTT assay was carried out according to the modified procedure for polymeric films (Nair et al 1997). Briefly, the extracts were used in preparing the culture medium and introduced into at least 12 wells of a 96 well tissue culture plate in which monolayer of mature L929 fibroblasts were grown. After $24 \mathrm{~h}$ incubation, the supernatant in each well was replaced with $50 \mu \mathrm{l}$ of MTT $(10 \mathrm{mg} / \mathrm{ml}$ in sterile phosphate buffered saline) and incubated for a further $4 \mathrm{~h}$. The blue formazan product formed by the reduction of the MTT by the live cells was measured spectrophotometrically $(570 \mathrm{~nm})$ to assess the cytotoxicity and/or activity of the cells.

2.3b Direct contact test: Cytotoxicity was also assessed by the direct contact test. PET film strips $(10 \times 5 \mathrm{~mm})$ were fixed onto tissue culture petri dishes by a Silastic medical grade adhesive (Dow Corning) and the growth of L929 fibroblasts in contact with films was compared microscopically to the controls.

\subsection{In vivo studies}

The PET films sterilized for the different cycles were intramuscularly implanted in black hooded Lister rats (Liverpool strain) for 1 month. After explantation, the tissue around the implant was immunostained (Vince et al 1991) using monoclonal antibodies for the macrophages (ED2) to specifically stain the macrophages. Specific stains for T cells, B cells and neutrophils were also used. The cell number and distribution were estimated using a computer aided image analysis system.

\section{Results and discussion}

Commercial polyethylene terephthalate (PET) is known to contain cyclic and linear oligomers as natural impurities which are formed as byproducts during polymerization 
and texturing. The content of cyclic trimer, a major oligomer component, is actually a criterion for the quality of PET. Studies of Nair and Sreenivasan (1984) and Nair (1990) on the morphological changes induced in PET on repeated steam sterilization revealed the degradation of amorphous regions and increase of cyclic trimer on the surface of the PET. While the total oligomer content may vary for PET samples from different sources, the studies of Nair and Sreenivasan (1984) and Nair (1990) have shown that the surface oligomer content increases on repeated steam sterilization.

Scanning electron micrographs of the surface of the polyester films which were subjected to different cycles of steam sterilization are presented in figures $1 \mathrm{a}-\mathrm{d}$. The bare surface of the polyester which was initially cleaned by soxhlet extraction with the solvents (methanol, water, ether) and further wiped with methanol, shows hardly any
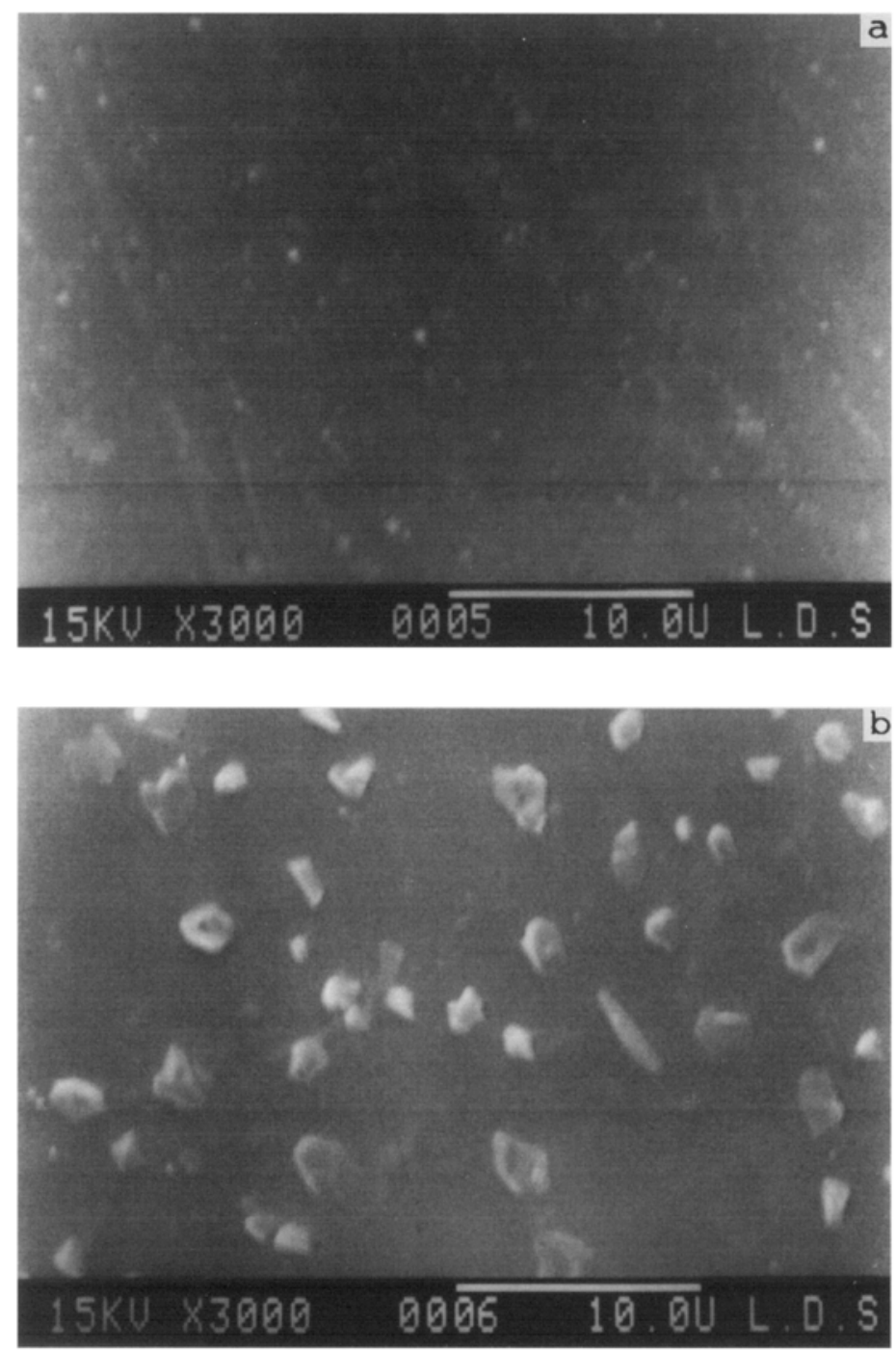

Figure 1. a. b. 

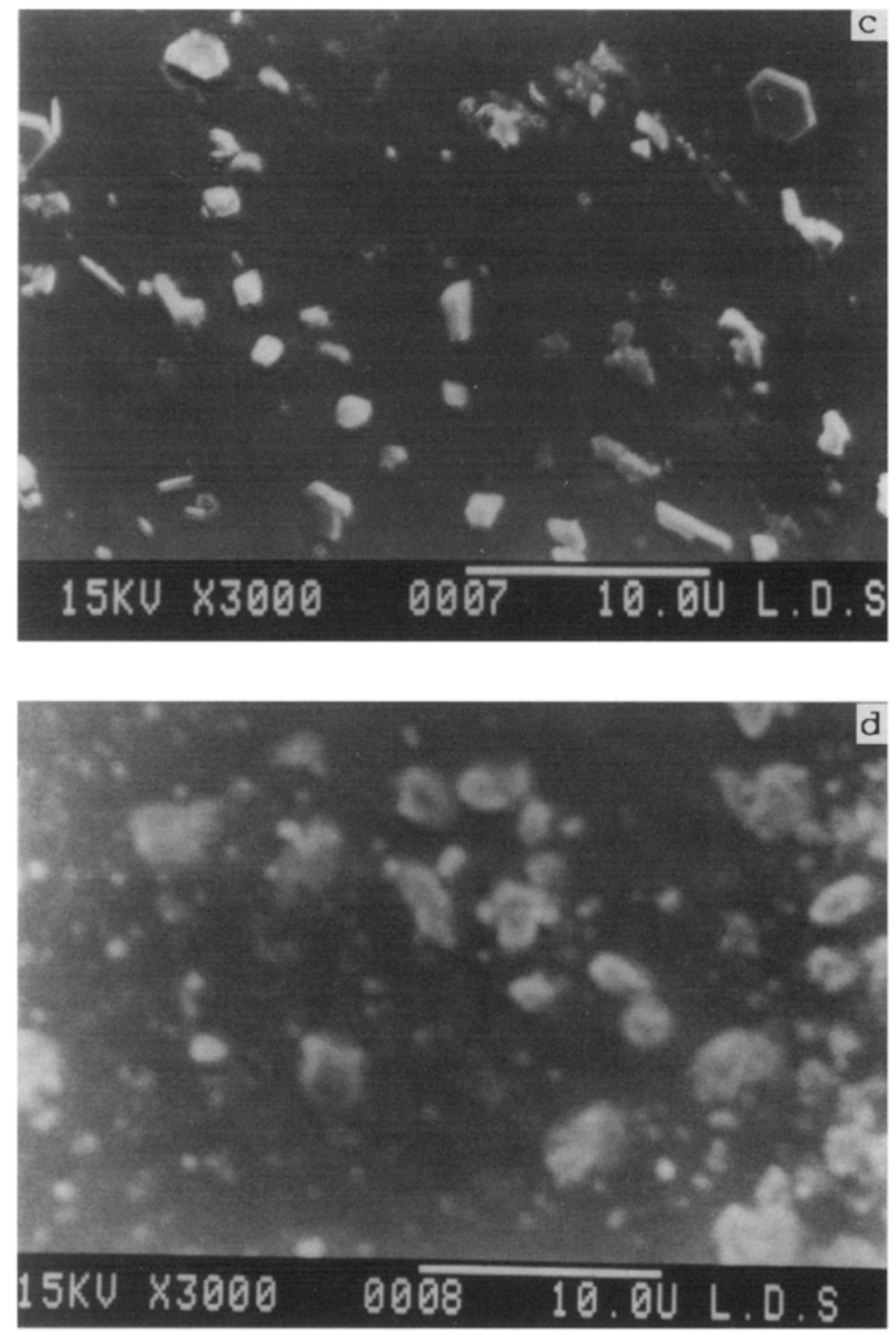

Figure 1. Scanning electron micrograph of the surface of PET film (a) bare, (b) steam sterilized for $15 \mathrm{~min}$, (c) steam sterilized for $30 \mathrm{~min}$ and (d) steam sterilized for $60 \mathrm{~min}$.

oligomers. However, when the same films were autoclaved, the oligomer content is seen to progressively increase with each cycle of steam sterilization.

\subsection{Influence of the surface oligomer on biocompatibility}

In this study, the MTT assay did not indicate significant cytotoxicity of the aqueous extracts, presumably due to the inability of water to efficiently extract the oligomers. The cells were directly grown on the films in the direct contact test. The cells which were 
spindle shaped and in a uniform monolayer in the control petridish, showed differing responses to each of the materials tested. Figure $2 a$ is the control plate consisting of a uniform layer of L929 fibroblasts. The PET sample autoclaved for the normal cycle of $15 \mathrm{~min}$ did not cause any changes in cell viability (figure $2 \mathrm{~b}$ ). When the cells were exposed to PET sterilized for $30 \mathrm{~min}$, some of the cells especially those which were immediately on the polymer surface lost their spindle shape and became rounded, some showing lysis. Uniform destruction of the cells is not observed indicating that the oligomer formation is in localized region as is clearly seen in the SEM (figure 1c). A zone of viable and normal cells is also observed in the periphery (figure $2 \mathrm{c}$ ). The cytotoxic nature of the oligomers is thus evident at higher concentrations of the same.
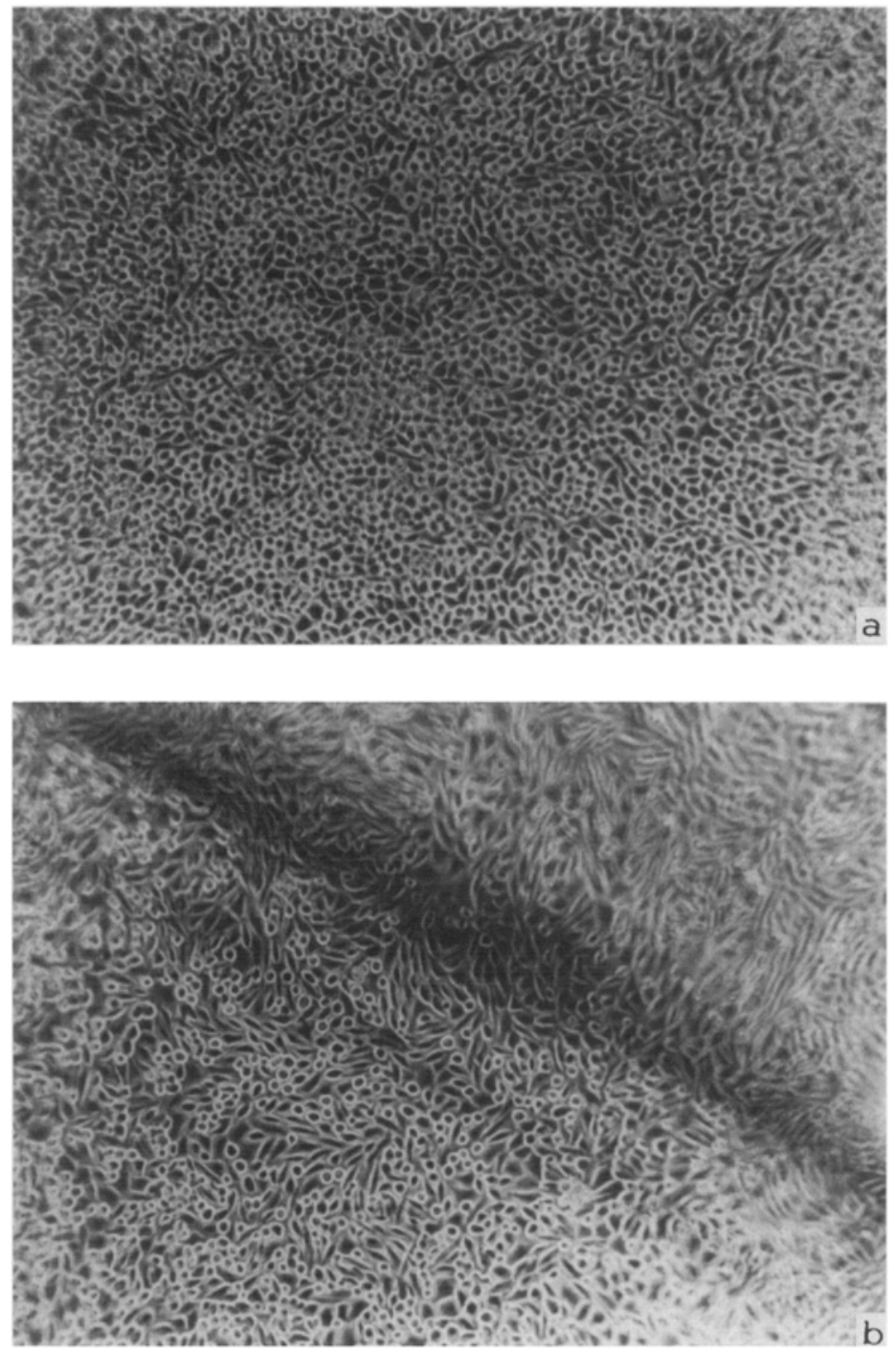

Figure 2. $\mathbf{a} \cdots \mathbf{b}$. 

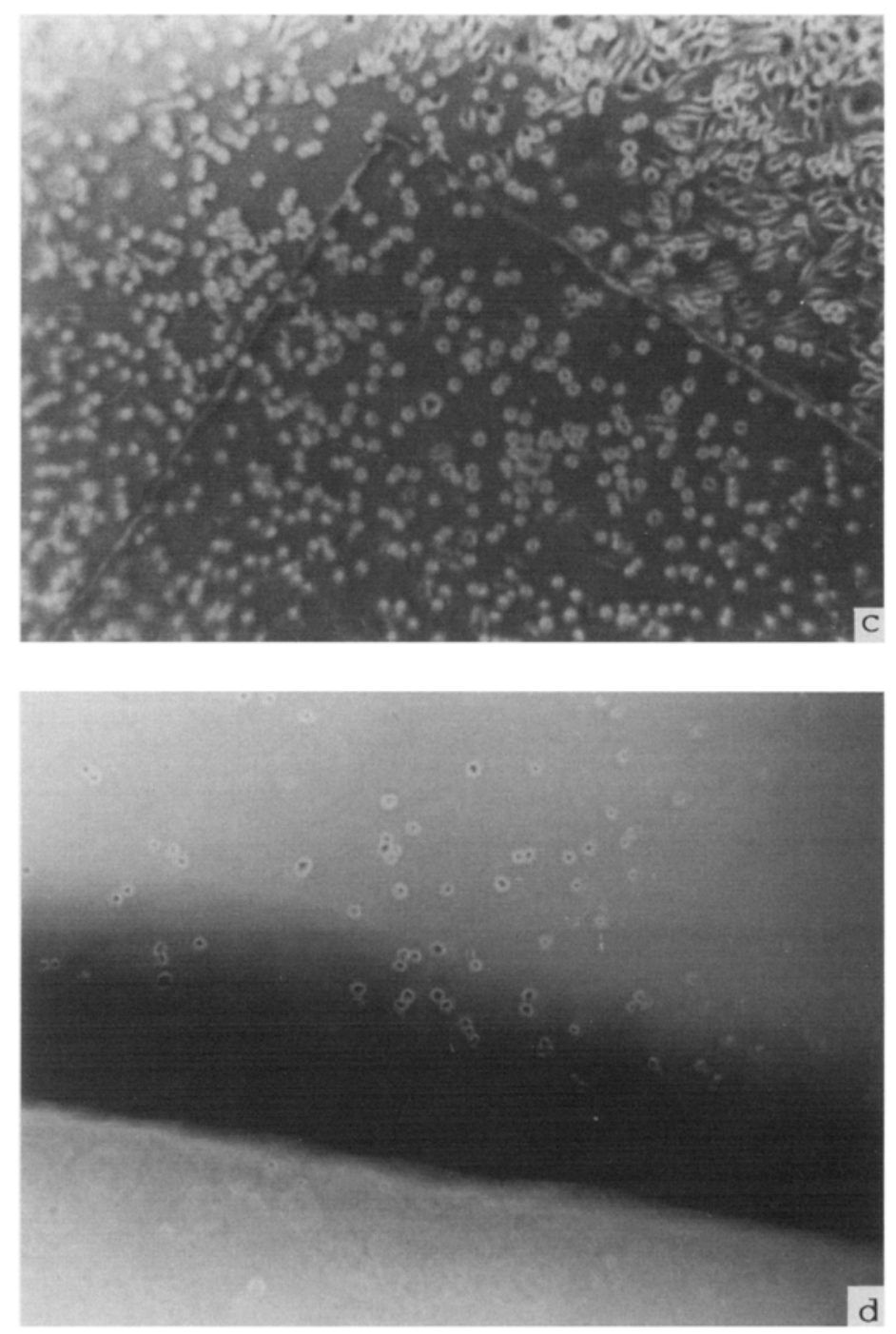

Figure 2. L929 fibroblast cell culture on different samples (a) control cells, (b) on PET film autoclaved for $15 \mathrm{~min}$, (c) on PET film autoclaved for $30 \mathrm{~min}$ and (d) on PET film autoclaved for $60 \mathrm{~min}$.

\subsection{In vivo studies}

Sawyer et al (1979) observed that the physiological responses (such as healing, intramural thrombus formation, structure and characterization of the inner and outer fibrous capsule) of porous polymeric dacron prostheses are influenced by the final cleaning, crimping and chemical procedures. Gumarglieva et al (1982) also suggested that the rapid disintegration of implants under conditions of infection is due to a chemotactic mechanism involving oligomers. In this study, the tissue response to the PET samples sterilized for the various time periods was assessed by the immunohistochemical staining method (Vince et al 1991). No T cells, B cells or neutrophils were 
Table 1. Effect of autoclaving on the tissue response of PET.

\begin{tabular}{lc}
\hline Time of autoclaving (min) & $\begin{array}{l}\text { Total number of ED2 macrophages at } \\
\text { implant site after } 1 \text { month }\end{array}$ \\
\hline 15 & 61 \\
30 & 152 \\
60 & 487 \\
\hline
\end{tabular}

observed at the implant site at the end of one month. A modified avidin-biotin technique using monoclonal antibody for ED2 macrophages was used to identify and quantify the macrophages. The monoclonal antibody specifically recognizes a membrane antigen present on the tissue macrophages of the rat. Monocytes, dendritic cells, lymphocytes and granulocytes are negative for ED2. An automated image analysis system was utilized to measure the cell number, cell area and distance from the implant. The cell numbers presented as a function of distance from the implant surface is shown in figure 3. The total number of cells at the implant site of each of the PET films are given in table 1. The total number of cells was counted over a 20 frame area, corresponding to a defined location in the section. The tissue response to the PET sample autoclaved for $15 \mathrm{~min}$, produced the minimum number of cells $(61)$ at the end of one month. There is an increase in the number of macrophages (152) when the $30 \mathrm{~min}$ sterilized PET sample was analysed in the same conditions. The maximum number of macrophages (487) was observed at the implant site of the 60 min sterilized PET sample.

Figure 3 also depicts the distribution of cells around the vicinity of the implant site, with a distance of 0 indicating the periphery of the implant. The ED2 macrophages around the PET sample sterilized with the normal cycle (1) are lesser in number with counts of 3.5 from the periphery of the implant up to a distance of $312 \mu$. The area beyond $312 \mu$ shows an absence of the ED2 macrophage counts except for a few macrophages at a distance of $624 \mu$ from the implant site. The tissue reaction is hence comparable to that of a normal wound healing. A higher number of macrophages with counts ranging from 3.5 to 14 can be seen up to a distance of $312 \mu$ in the case of the PET implant which was sterilized in the $30 \mathrm{~min}$ cycle. The area beyond $312 \mu$ shows ED2 macrophages with a lesser count of 2 . The 60 min sterilized samples seemed to elicit an even distribution with uniformly higher numbers of cells and counts ranging from 4 to 20 up to a distance of $640 \mu$ from the implant site. The higher number of oligomers produced in the $30 \mathrm{~min}$ and $60 \mathrm{~min}$ cycles in comparison to the normal cycle may be responsible for the enhanced macrophage activity for these samples.

One of the primary roles of the macrophages is to engulf foreign debris and phagocytose them. The increased oligomers may therefore be inducing the macrophages to phagocytose and to detoxify the materials. The increased macrophage number also denotes lesser biocompatibility for the sample sterilized in the $30 \mathrm{~min}$ and $60 \mathrm{~min}$ cycles. A long term effect of such phagocytosis may adversely affect the integrity of the polyester material by causing accelerated degradation of the matrix material. 


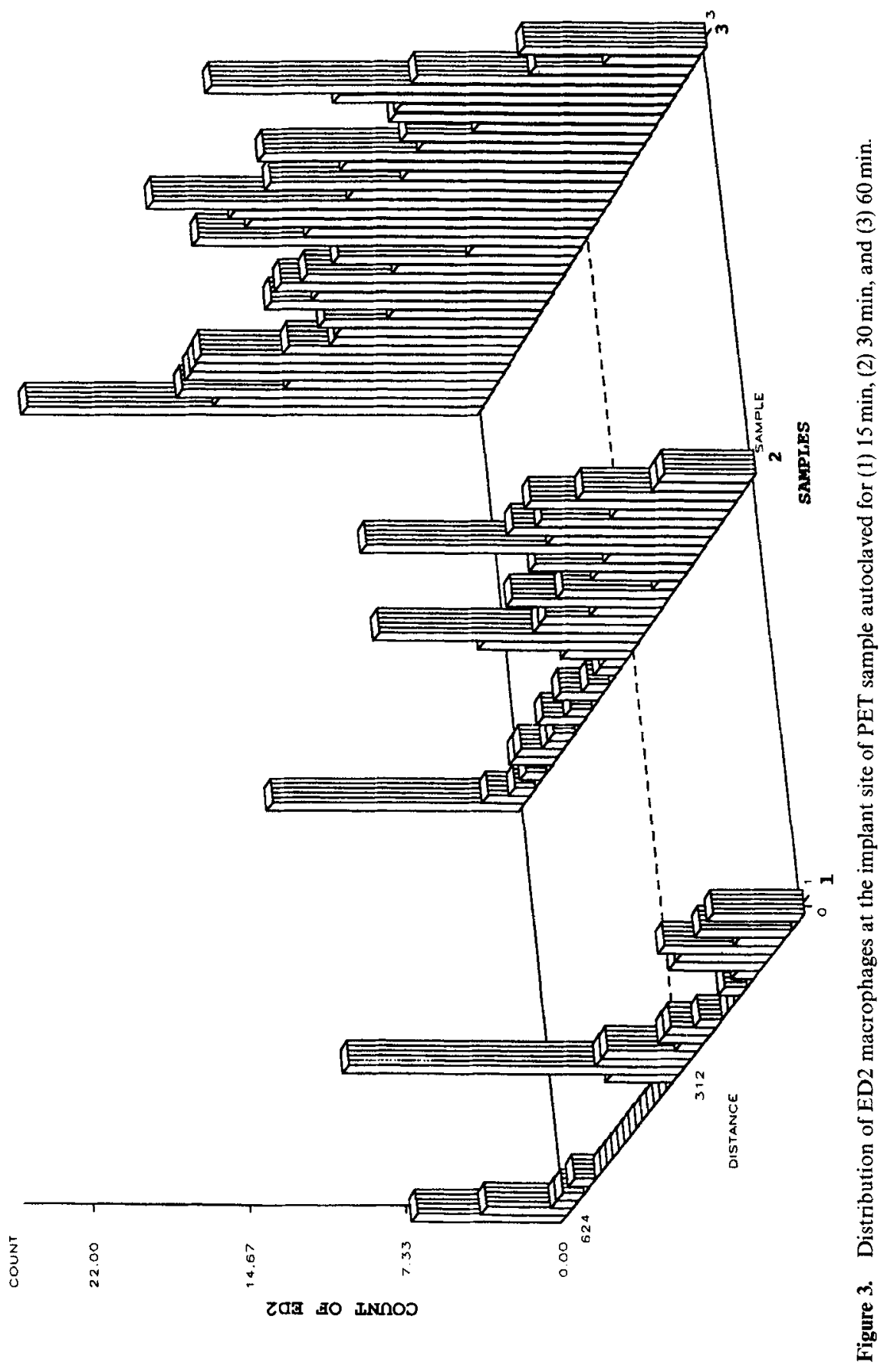




\section{Conclusions}

Repeated steam sterilization of PET samples is seen to affect the biocompatibility and possible long term stability of the materials. It may therefore be desirable to avoid the repeated steam sterilization of polyester materials especially for long term implant applications, if stability of the material is a matter of concern. The study also highlights the need for the evaluation of each material specifically with relevance not only to the sterilization method but also to the period of the sterilization employed.

\section{Acknowledgement}

One of the authors (PDN) is grateful to DST, New Delhi, for the award of a BOYSCAST Fellowship at Univ. of Liverpool, UK.

\section{References}

Conway P F, Simmens S C and Charlesworth D 1979 Br. J. Surg. 66262

Gumarglieva K Z, Moiseev Yu V, Daurova T T and Voronka D S 1982 Biomat. 3177

Haberman V and Waitzova D 1985 Arch. Toxicol. Suppl. 8458

Nair P D 1990 Clin. Mat. 543

Nair P D, Doherty P J and Williams D F 1997 Mater, in Med. (in press)

Nair P D and Sreenivasan K 1984 Biomat. 5305

Sawyer P N, Stanczewski B, Hoskin G P. Sophie Z, Stilman R M, Turner R J and Hoffman H L $1979 \mathrm{~J}$. Biomed. Mater. Res. 13937

Vince D G, Hunt J and Williams D F 1991 Biomat. 12731 